\title{
Estimativa de Parâmetros em um Sistema de Leito Móvel Simulado via Algoritmo de Colisão de Partículas Populacional
}

\section{Parameter Estimation of a Simulated Moving Bed System via Population Particle Collision Algorithm}

Claudir Oliveira1, Jader Lugon Junior², Wagner Figueiredo Sacco³, Diego

Campos Knupp ${ }^{4}$, Antônio José da Silva Neto ${ }^{4}$

\section{RESUMO}

Dadas as inúmeras aplicações importantes do Leito Móvel Simulado nas diferentes áreas das engenharias, verifica-se a necessidade na determinação e ajustes de parâmetros para a modelagem matemática do problema de interesse. Este trabalho tem por objetivo a determinação de parâmetros cromatográficos envolvidos na separação dos enantiômeros em uma unidade cromatográfica de leito móvel simulado. Para estimativa de parâmetros de transferência de massa utilizou-se o algoritmo de Colisão de Partículas Populacional. Na solução do problema inverso empregou-se a abordagem da Máxima Verossimilhança e a estimativa por Maximum a Posteriori (MAP). Com a aplicação das abordagens selecionadas, combinadas com as técnicas de discretização e o modelo de coluna empregado, foi possível descrever adequadamente os fenômenos envolvidos no transporte de massa.

Palavras-chave: Problemas Inversos. Determinação de Parâmetros. Físico-Químicos. Método Estocástico.

\section{ABSTRACT}

Given the numerous important applications of the Simulated Mobile Bed in the different areas of engineering, there is a need in the determination and adjustments of parameters for the mathematical modeling of the problem of interest. This work aims to determine the chromatographic parameters involved in the separation of the enantiomers in a simulated moving bed chromatographic unit. For the estimation of mass transfer parameters, the Population Particle Collision algorithm was used. In the solution of the inverse problem, the Maximum likelihood approach was used as well as Maximum a Posteriori (MAP). With the application of the approaches considered, combined with the discretization techniques and the column model employed, it was possible to adequately describe the mass transport phenomena involved.

Keywords: Inverse Problems. Determination of Parameters. Physical-Chemical. Stochastic Method.
${ }^{1}$ Doutor em Modelagem Computacional. Universidade Federal do Oeste do Pará UFOPA

E-mail:

claudir.oliveira@ufopa.edu.br

${ }^{2}$ Doutor em Modelagem

Computacional. Instituto Federal Fluminense - IFF/Campus Macaé RJ.

${ }^{3}$ Doutor em Modelagem Computacional. Universidade Federal do Oeste do Pará UFOPA

${ }^{4}$ Doutor em Engenharia Mecânica. Instituto Politécnico - UERJ. 


\section{INTRODUÇAOO}

A cromatografia vem sendo desenvolvida como um dos principais processos de separação, especialmente o Leito Móvel Simulado (LMS) e que tem sido objeto de estudo em diversos trabalhos na literatura. Neste trabalho aplicou-se a solução de problemas inversos em um fenômeno de transferência de massa usando Leito Móvel Simulado (LMS) visando o cálculo de parâmetros dos fenômenos envolvidos em um processo de separação de substâncias. Com este propósito, o método de Linha é usado para a discretização espacial das equações e o método de Leapfrog foi usado como técnica de integração e implementada para ser usada como solução numérica do modelo de transferência de massa ao longo do tempo. Na implementação da rotina computacional para a modelagem matemática do LMS foi usado o modelo de Transporte Dispersivo e em seguida foi realizada a simulação de separação dos enantiômeros 1,1'-bi-2-naftol.

O bi-naftol é um dos constituintes mais procurados em separação quiral e é uma das aplicações bastante usadas na tecnologia de LMS e que tem sido objeto de estudos por diversos pesquisadores como, PAIS et al. (1998); WONGSO e HIDAJAT (2004); LI e KAWAJIRI (2011); KAWAJIRI e BIEGLER (2005; 2008).

Duas abordagens são utilizadas para a determinação dos parâmetros com o problema inverso. A primeira abordagem consiste no uso da estimativa da Máxima Verossimilhança (Maximum Likelihood Estimation - MLE) e na segunda abordagem utilizou-se a Estimativa por Maximum a Posteriori (MAP). Para ambas abordagens, utilizou-se uma versão variante do Algoritmo de Colisão de Partículas (Particle Collision Algorithm - PCA) denominada de Algoritmo de Colisão de Partículas Populacional (SACCO et al. 2007). Nessa variante o método usa um número maior de partículas, diferenciando-se da versão canônica do PCA que usa apenas uma partícula.

\section{MODELAGEM COMPUTACIONAL}

Um sistema de leito móvel simulado consiste em um conjunto de colunas cromatográficas, com válvulas que controlam e alternam os fluxos de entrada e saída. Em sua modelagem são empregadas as equações para o balanço de massa nos nós do sistema, que conectam às colunas de acordo com configurações específicas. Dos modelos clássicos que representam a modelagem de colunas em sistemas de LMS, é adotado neste trabalho o modelo de Transporte Dispersivo por ter se mostrado com bom compromisso entre precisão e simplicidade, de acordo com BIEGLER e JIANG (2004), e por ser 
considerado como eficiente na reprodução dos perfis de concentração. O modelo de transporte dispersivo é baseado na aplicação da primeira lei de Fick de transporte de massa e é usado neste trabalho para descrever o balanço de massa entre as fases líquida e sólida ao longo de uma coluna em cromatografia líquida. $O$ modelo para ambas as fases pode ser escrito pelas seguintes equações,

$$
\begin{gathered}
\frac{\partial C_{i, k}}{\partial t}+\left(\frac{1-\varepsilon_{b}}{\varepsilon_{b}}\right) \frac{\partial q_{i, k}}{\partial t}=D_{a x, l} \frac{\partial^{2} C_{i, k}}{\partial x^{2}}-v_{l} \frac{\partial C_{i, k}}{\partial x}, i=A, B, k=1, \ldots, N_{c}, l=1, \ldots, 4 \\
\frac{\partial q_{i, k}}{\partial t}=k_{e f f, i}\left(q_{i, k}^{e q}-q_{i, k}\right)
\end{gathered}
$$

onde $\varepsilon_{b}$ é a porosidade e representa a fração da coluna não ocupada por partículas, $D_{a x}$ é o coeficiente de dispersão axial, $v$ é a velocidade da fase líquida, $k_{e f f, i}$ é o coeficiente de transferência de massa, $q_{i}^{e q}$ é a concentração de equilíbrio, ou seja, é a quantidade adsorvida de cada enantiômero na fase estacionária equilibrada com a fase móvel, $C_{i}$ e $q_{i}$ representam as concentrações das duas componentes $(i=A, B)$ nas fases líquida e sólida, respectivamente, nas $N_{c}$ colunas de cada seção $l$ do Leito Móvel Simulado.

A isoterma bi-Langmuir é usada neste trabalho para descrever o comportamento adsortivo. Este modelo competitivo para o sistema de LMS é descrito pela Equação 3 (YAO et al., 2008), escrita em função de suas componentes $i$ para cada coluna $k$ sendo dadas então por:

$$
q_{A, k}^{e q}=\frac{H_{i, 1} C_{i, k}}{1+K_{A, 1} C_{A, k}+K_{B, 1} C_{B, k}}+\frac{H_{i, 2} C_{i, k}}{1+K_{A, 2} C_{A, k}+K_{B, 2} C_{B, k}}
$$

onde, $H_{i, 1}$ e $H_{i, 2}$ são as capacidades de saturação dos dois sítios, $K_{i, 1}$ e $K_{i, 2}$ são as constantes de equilíbrio dos sítios para as componentes $i=A, B$.

As condições iniciais, para ambas as fases, considerando uma coluna $k$ livre de qualquer espécie $i$ no início da operação, foram as mesmas consideradas em SILVA (2000) e as condições de contorno na fase líquida entre as correntes de extrato e rafinado, dentro de uma seção, são representadas por,

$$
C_{i, k}\left(t, x_{k}=0\right)=C_{i, k-1}\left(t, x_{k-1}=L_{c}\right), k=2, \ldots, N_{c}
$$


e entre as correntes de dessorvente e alimentação, as condições são representadas pelas seguintes equações,

$$
\begin{gathered}
C_{i, 1}\left(t, x_{1}=0\right)=C_{i, N_{c}}\left(t, x_{N_{C}}=L_{c}\right) \frac{v_{4}}{v_{3}} \\
C_{i, 5}\left(t, x_{5}=0\right)=\frac{v_{2} C_{i, 4}\left(t, x_{4}=L_{c}\right)+v_{f} C_{i, f}}{v_{3}}
\end{gathered}
$$

onde $v_{f}$ é a velocidade de alimentação e $C_{i, f}$ é a concentração da componente injetada.

Na saída de cada coluna, as equações de advecção são usadas conforme sugerido em HAAG et al. (2001) dada pela Equação 7,

$$
\frac{\partial C_{i, k}}{\partial t}=v_{l} \frac{\partial C_{i, k}}{\partial x_{k}}
$$

e na fase sólida a condição é dada por,

$$
q_{i, k}\left(t, x_{k}=L_{c}\right)=q_{i, k+1}\left(t, x_{k+1}=0\right)
$$

\subsection{Formulação e solução do problema direto}

Com a solução do problema direto foram obtidas as respostas do modelo associadas ao fenômeno de transporte de massa. Essas respostas foram utilizadas no processo do problema inverso visando identificar os parâmetros do modelo que melhor descrevem os dados experimentais disponíveis.

A técnica numérica usada para resolução das equações diferenciais parciais (EDPs) consistiu no Método de Linhas (MOL). A discretização da Equação 1 foi aplicada apenas para a aproximação espacial e deixando contínua a variável no tempo. Neste procedimento as EDPs foram resolvidas pela técnica de diferenças finitas e as equações de equilíbrio foram transformadas em equações diferenciais ordinárias. O termo difusivo é discretizado então empregando-se a técnica de diferenças finitas centradas de segunda ordem nos nós internos, resultando na seguinte expressão numérica,

$$
\frac{\partial^{2} C_{i, k}}{\partial x^{2}}=D_{a x}\left(\frac{C_{i, k}^{n, j-1}-2 C_{i, k}^{n, j}+C_{i, k}^{n, j+1}}{\Delta x^{2}}\right), j=1, \ldots, N-1
$$

e o termo advectivo passa a ter uma primeira ordem, pela fórmula de diferença centrada com dois pontos, 
Oliveira, C.; Lugon Jr., J.; Sacco, W. F.; Knupp, D. C., Silva Neto, A. J. Estimativa de Parâmetros em um Sistema de Leito Móvel Simulado via Algoritmo de Colisão de Partículas Populacional

$$
\frac{\partial C_{i, k}}{\partial x}=-v_{l}\left(\frac{C_{i, k}^{n, j+1}-C_{i, k}^{n, j-1}}{2 \Delta x}\right)
$$

Para a solução do sistema de EDOs, empregou-se o método de Leapfrog. A essência do método consiste em atualizar as concentrações em tempos intermediários àqueles em que são atualizadas as posições, isto é, ao invés de atualizar as posições e as concentrações no mesmo instante $t_{1}=t+\Delta t$, primeiro atualiza-se a posição no instante $t+\Delta t$. Com base na concentração $C_{1 / 2}$ de $t_{1 / 2}=t+\frac{\Delta t}{2}$ a concentração $C_{3 / 2}$ é calculada em $t+\frac{3 \Delta t}{2}$. A nova concentração de cada passo é então atualizada em $t+\frac{\Delta t}{2}$ sempre com base na concentração de $t-\frac{\Delta t}{2}$ e assim sucessivamente, de modo que as posições e concentrações vão saltando ao longo do tempo, de forma intercalada.

Os perfis de concentração calculados com a solução do problema direto foram validados com dados experimentais obtidos da literatura e são apresentados na Figura 1.

a)

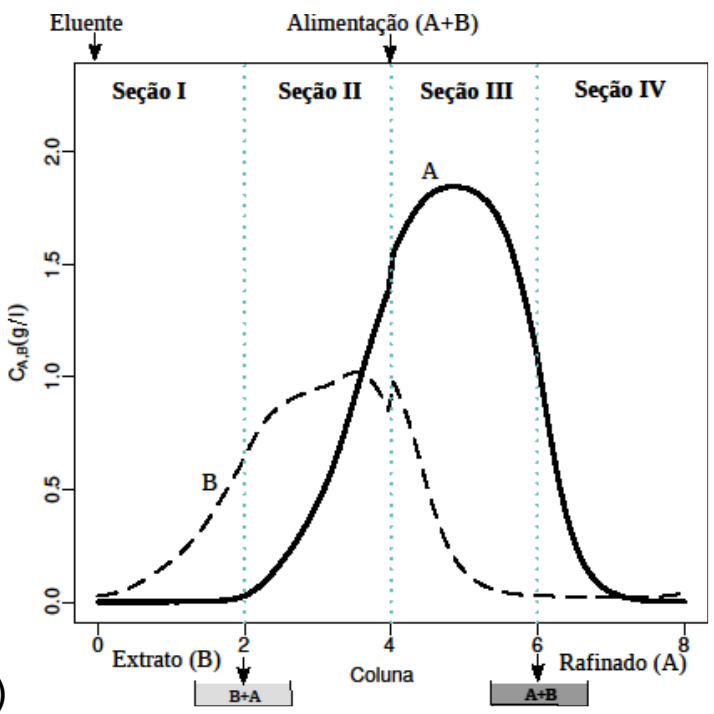

b)

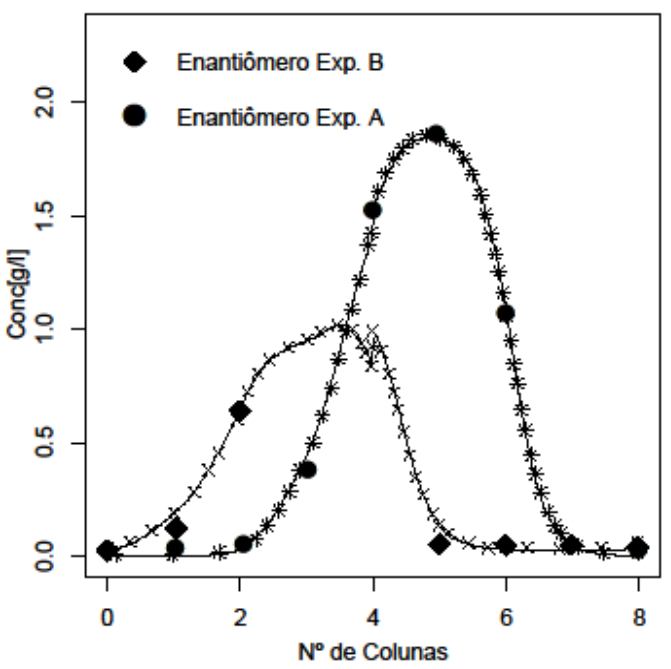

Figura 1. a) Perfil interno de concentração dos isômeros ao longo das colunas do LMS e b) comparação com os dados experimentais

Na Figura 1 os símbolos $\bullet$ e representam os pontos experimentais para os isômeros mais adsorvido $(B)$ e menos adsorvido $(A)$, respectivamente; $\times e^{*}$ representam os valores de referência, calculados por PAIS et al. (1998) e as linhas contínuas representam os dados calculados com os esquemas adotados neste trabalho. 
O problema inverso consiste em encontrar uma variável $x$, relacionada a uma observação $y$ escrita na forma $y=f\left(x, \varepsilon_{Y}\right)$, onde $y \in \mathfrak{R}^{M}$ representa a quantidade medida, $x \in \mathfrak{R}^{n}$ é a quantidade que se procura obter informações, $\varepsilon_{Y}$ representa o erro associado às medições do ruído e às imprecisões contidas no modelo e $f: \Re^{n} \times \Re^{m} \rightarrow \mathfrak{R}^{M}$ é o modelo. Os ruídos para este trabalho foram simulados nos dados experimentais reais existentes usando a Equação 11,

$$
\mathbf{Y}=\tilde{\mathbf{Y}}+\varepsilon_{\tilde{Y}^{\prime}}, \quad \varepsilon_{\tilde{Y}} \sim N\left(0, \sigma_{\tilde{Y}}\right)
$$

onde $\tilde{Y}$ corresponde aos dados experimentais reais e $Y$ é o vetor dos dados experimentais com ruídos simulados.

Com o propósito de avaliar as incertezas neste trabalho utilizou-se diferentes conjuntos de dados experimentais. A ideia com esta abordagem é tentar obter as incertezas tanto por parte da estocasticidade do método quanto devido ao ruído experimental. Para a simulação dos ruídos nos dados experimentais foram considerados $\varepsilon_{\tilde{Y}}=1 \%, 5 \%$ e $10 \%$ do valor máximo de $\boldsymbol{Y}$.

Para a solução do problema inverso primeiramente será usado o procedimento da máxima verossimilhança, que resulta em um problema de otimização, onde a função objetivo, que caracteriza os desvios entre dados experimentais e as previsões do modelo deve ser minimizada. Na outra, a abordagem consiste no uso do Maximum a posteriori, em que se considera haver alguma informação a priori sobre algum parâmetro de interesse.

\subsection{Procedimento da Máxima Verossimilhança}

A Estimativa por Máxima Verossimilhança (Maximum Likelihood Estimation - MLE) é um procedimento usado para estimar parâmetros de modelos estatísticos a partir do ajuste de dados do modelo. A função objetivo é a medida da distância entre o modelo calculado e os dados experimentais. Quando se admite que o modelo é perfeito e as flutuações dos erros experimentais são conhecidas e com distribuição normal, tem-se a função de densidade de probabilidade dos desvios com relação ao experimento, que deve ser maximizada. A função, neste caso, é dada pela Equação 12, conforme KAIPIO e SOMERSALO (2005). 


$$
p(\mathbf{Y} \mid \mathbf{Z})=\frac{1}{\sqrt{(2 \pi)^{N_{d}}}}|\mathbf{W}|^{-\frac{1}{2}} \times \exp \left(-\frac{1}{2}[\mathbf{Y}-\mathbf{C}(\mathbf{Z})]^{T} \mathbf{W}^{-1}[\mathbf{Y}-\mathbf{C}(\mathbf{Z})]\right)
$$

onde $\mathbf{Y}$ e C são vetores correspondentes aos dados experimentais e calculados, respectivamente, $\mathbf{Z}$ é o vetor de parâmetros, $\boldsymbol{W}$ é a matriz de covariância dos erros experimentais e $N_{d}$ é o número de dados experimentais.

Para se obter as estimativas de máxima verossimilhança, o problema inverso é formulado implicitamente resultando em um problema de otimização, onde deseja-se minimizar o funcional de resíduos quadrados entre as quantidades medidas experimentalmente e os valores calculados pelo modelo. A função objetivo, portanto, utilizada no procedimento de inversão é dada pela Equação 13, a qual resulta em parâmetros com variância mínima,

$$
R(\mathbf{Z})=\left[Y_{i}-C_{i}(\mathbf{Z})\right]^{T}\left[Y_{i}-C_{i}(\mathbf{Z})\right]=\sum_{i=0}^{N_{C}}\left[Y_{i}-C_{i}(\mathbf{Z})\right]^{2}
$$

\subsection{Estimativa por Maximum a Posteriori - MAP}

Supondo existir informação a priori para os parâmetros na forma de uma distribuição Gaussiana, e que Y e $\mathbf{Z}$ são independentes, utiliza-se a estimativa do Maximum a posteriori para o procedimento de minimização da função objetivo. A partir do teorema de Bayes temse,

$$
p(Z \mid Y)=\frac{p(Y \mid Z) q(Z)}{p(Y)}
$$

que corresponde à densidade de probabilidade de se observar as amostras quando o parâmetro é igual a Z. Aplicando a função $\ln ($. ) na Equação 14,

$$
\ln (p(\mathbf{Z} \mid \mathbf{Y}))=\ln (q(\mathbf{Z}))+\ln (p(\mathbf{Y} \mid \mathbf{Z}))-\ln (p(\mathbf{Y}))
$$

onde,

$$
q(\mathbf{Z})=2 \pi^{\frac{-N_{u n}}{2}}\left|\mathbf{V}^{-\frac{1}{2}}\right| \times \exp \left(-\frac{1}{2}\left[\mathbf{Z}-\mu_{p r}\right]^{T} \mathbf{V}^{-1}\left[\mathbf{Z}-\mu_{p r}\right]\right)
$$

onde $\mu_{p r}$ é a informação a priori e $V$ representa a matriz de covariância.

$$
p(\mathbf{Y} \mid \mathbf{Z})=2 \pi^{-\frac{N_{d}}{2}}|\mathbf{W}|^{-\frac{1}{2}} \times \exp \left(-\frac{1}{2}[\mathbf{Y}-\mathbf{C}(\mathbf{Z})]^{T} \mathbf{W}^{-1}[\mathbf{Y}-\mathbf{C}(\mathbf{Z})]\right)
$$

Resolvendo os termos da Equação 16, obtém-se a Equação 18, 


$$
\begin{aligned}
\ln (p(\mathbf{Z} \mid \mathbf{Y}))= & \ln \left(2 \pi^{\frac{-N_{u n}}{2}}\right)+\ln \left(|\mathbf{V}|^{-\frac{1}{2}}\right)+\ln \left(\exp \left(-\frac{1}{2}\left[\mathbf{Z}-\mu_{p r}\right]^{T} \mathbf{V}^{-1}\left[\mathbf{Z}-\mu_{p r}\right]\right)\right) \\
= & -\frac{1}{2} N_{u n} \ln (2 \pi)+\frac{1}{2} \ln \left|\mathbf{V}^{-1}\right|+\left(-\frac{1}{2}\left[\mathbf{Z}-\mu_{p r}\right]^{T} \mathbf{V}^{-1}\left[\mathbf{Z}-\mu_{p r}\right]\right)+ \\
& -\frac{1}{2} N_{d} \ln (2 \pi)+\frac{1}{2} \ln \left|\mathbf{W}^{-1}\right|+\left(-\frac{1}{2}[\mathbf{Y}-\mathbf{C}(\mathbf{Z})]^{T} \mathbf{W}^{-1}[\mathbf{Y}-\mathbf{C}(\mathbf{Z})]\right) \\
= & -\frac{1}{2}\left[\left(N_{u n}+N_{d}\right) \ln (2 \pi)+\ln \left|\mathbf{V}^{-1}\right|+\ln \left|\mathbf{W}^{-1}\right|+F_{\text {map }}(\mathbf{Z})\right]
\end{aligned}
$$

onde

$$
F_{\text {map }}(Z)=\left([Y-C(Z)]^{T} W^{-1}[Y-C(Z)]+\left[Z-\mu_{p r}\right]^{T} V^{-1}\left[Z-\mu_{p r}\right]\right.
$$

O vetor $\mathbf{Z}$ que maximiza $\ln (p(\mathbf{Z} \mid \mathbf{Y}))$ é igual a $\max [p(Z j Y)]$, implicando na Equação 20,

$$
\max _{\mathbf{Z}} p(\mathbf{Z} \mid \mathbf{Y}) \Leftrightarrow \max _{\mathbf{Z}}[\ln (p(\mathbf{Z} \mid \mathbf{Y}))) \Leftrightarrow \min _{\mathbf{Z}}\left[F_{\text {map }}(\mathbf{Z})\right]
$$

onde $\mathbf{Z}$ corresponde ao vetor das amostras aleatórias com média $\mu$ e matriz de covariância conhecida $V$ que introduzem informação a priori a respeito do vetor $\mathbf{Z}$. A minimização de $F_{\text {map }}$ produz estimativas de $\mathbf{Z}$ que maximizam a distribuição a posteriori $p(\mathbf{Z} \mid \mathbf{Y})$.

\subsection{Algoritmo de Colisão de Partículas Populacional}

A versão populacional do Algoritmo de Colisão de Partículas PopPCA foi introduzida por SACCO et al. (2007) e aplicada a um problema de otimização que consiste em ajustar parâmetros de célula de um reator nuclear. Trata-se de uma hibridização do Algoritmo de Colisão de Partículas (PCA) e do Algoritmo Genético. A diferença é que na versão populacional, o algoritmo populacional é modificado com o acréscimo de um loop que incrementa sobre o número da população (especificamente sobre o número de partículas) e a chamada para uma função, denominada de NewPopulation, que calcula a população para a próxima geração.

De acordo com o referido autor, a motivação dessa modificação é promover uma maior exploração do espaço de busca. Os demais passos reproduzem exatamente a mesma estratégia do PCA, onde cada partícula se move no espaço de busca através de eventos de colisão sucessivas. O algoritmo apresentado na Figura 2 representa o esquema populacional introduzido no PCA para um processo de minimização. 


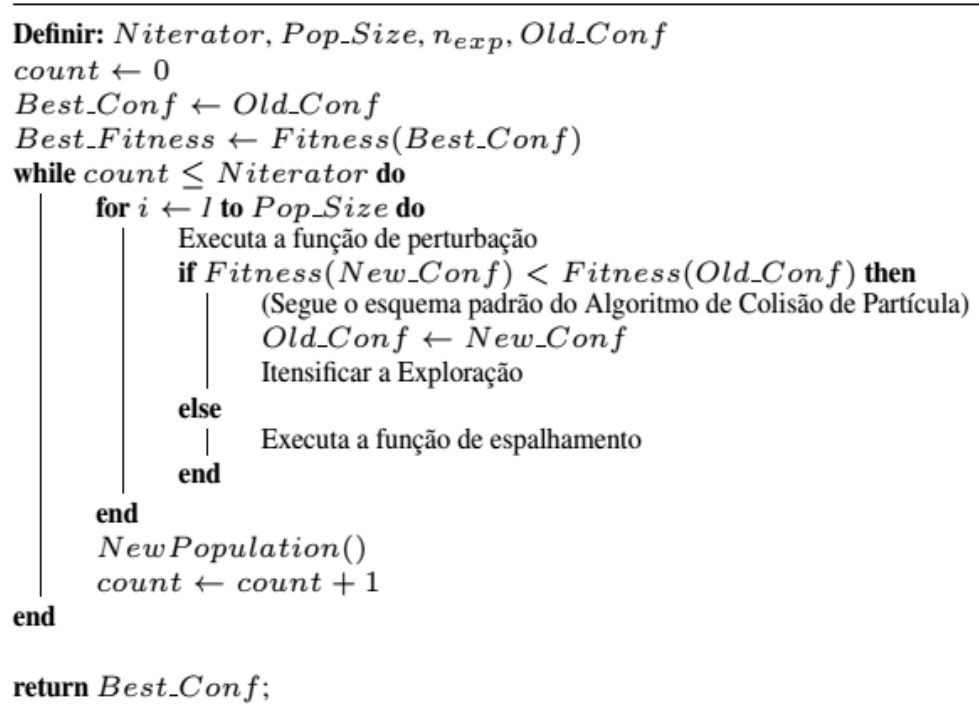

Figura 2. Algoritmo de Colisão de Partículas Populacional - PopPCA

Os candidatos à solução são emparelhados de forma aleatória, e um cruzamento é feito por meio de um processo de computação evolutiva. Nesse processo, escolhe-se mais de uma solução progenitora e produz-se uma solução descendente a partir delas. A técnica de "recombinação em um ponto" foi usada para produzir uma solução descendente. $\mathrm{Na}$ técnica, um ponto de corte é determinado aleatoriamente e os segmentos antes deste ponto são trocados. Depois disso, os "pais" e "irmãos" são classificados em ordem decrescente da função de adaptabilidade Fitness e os primeiros indivíduos deste conjunto ordenado sobrevivem para a próxima geração (SACCO et al. 2007).

\section{RESULTADOS E DISCUSSÖES}

Visando estudar a influência dos parâmetros que determinam os perfis de concentração das substâncias, realiza-se o estudo de sensibilidade dos parâmetros. Um coeficiente de sensibilidade $J_{i j}$, como definido na Equação 21, é uma medida da sensibilidade da concentração calculada $C_{i}$ com respeito às variações dos parâmetros do vetor $\mathbf{Z}$, i.e. é a derivada da concentração em relação aos parâmetros da isoterma, sendo calculada por (LUGON Jr., 2005),

$$
J_{i, j}=\frac{\partial C_{i}}{\partial Z_{j}}
$$

representando, portanto, a resposta do modelo matemático a uma variação finita no valor dos parâmetros conforme os gráficos da Figura 3. 

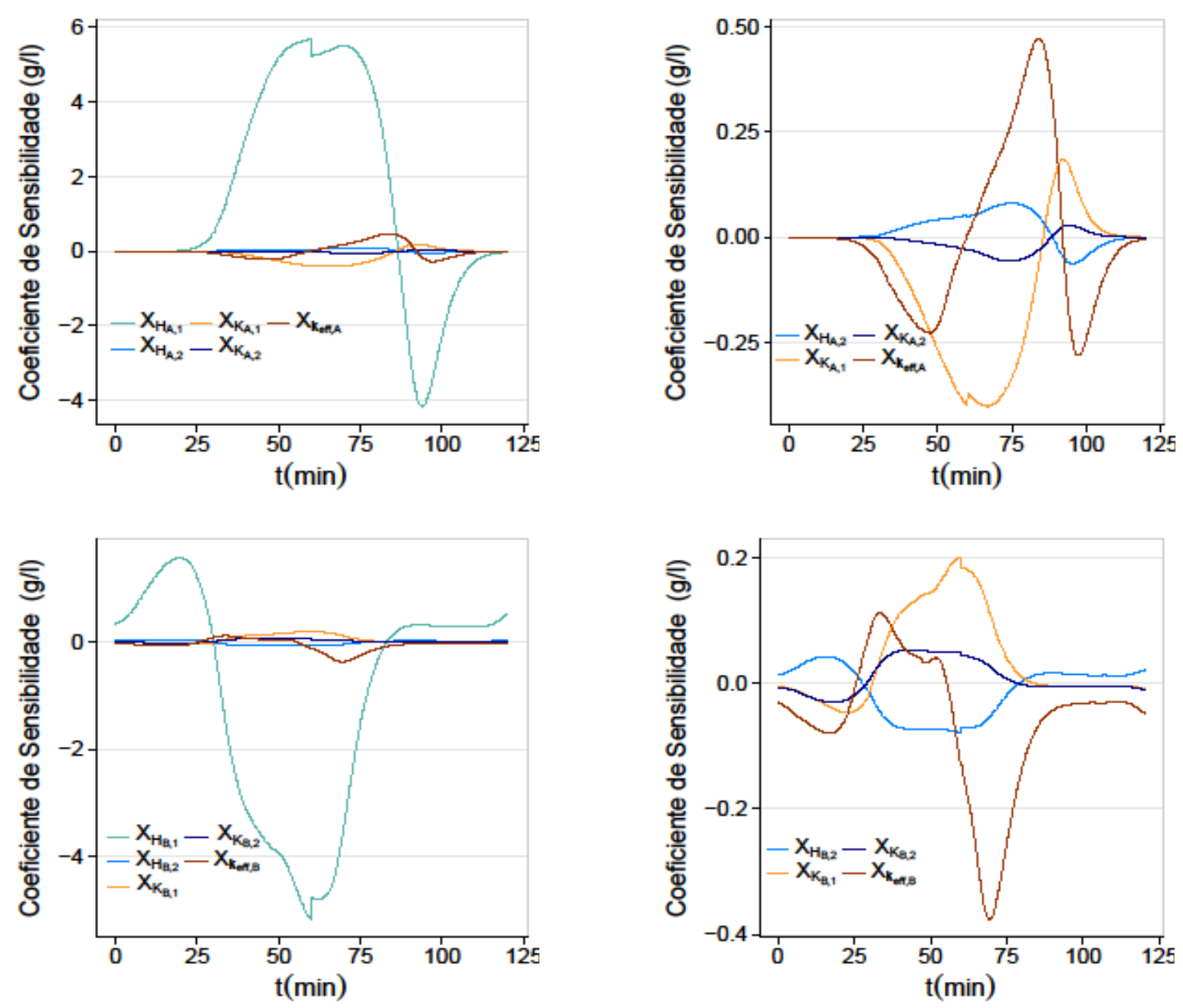

Figura 3. Análise de sensibilidade dos parâmetros da equação de isoterma de biLangmuir para as concentrações de $\mathrm{A}$ e $\mathrm{B}$

Com base nos resultados apresentados pela análise de sensibilidade realizou-se as buscas das estimativas por meio dos procedimentos da máxima verossimilhança (MLE) e do Maximum a posteriori (MAP). Foram realizadas 30 execuções com o método PopPCA, estipulando-se um número de avaliações para a função objetivo em cada execução. Os intervalos de busca dos parâmetros usados durante a minimização da função objetivo para as duas estratégias (MLE e MAP) são apresentados na Tabela 1. São apresentados também os valores de referência de acordo com PAIS et al. (1998). 
DOI 10.18605/2175-7275/cereus.v10n3p120-135
Oliveira, C.; Lugon Jr., J.; Sacco, W. F.; Knupp, D. C., Silva Neto, A. J. Estimativa de Parâmetros em um Sistema de Leito Móvel Simulado via Algoritmo de Colisão de Partículas Populacional

Tabela 1. Intervalos de busca dos parâmetros usados no PopPCA e valores de referência.

\begin{tabular}{ccc|ccc}
\hline Param. $\left(C_{A}\right)$ & Valor Ref. & {$\left[\boldsymbol{L}_{\boldsymbol{m i n}} ; \boldsymbol{L}_{\boldsymbol{m a x}}\right]$} & Param. $\left(\boldsymbol{C}_{\boldsymbol{B}}\right)$ & Valor Ref. & {$\left[\boldsymbol{L}_{\boldsymbol{m i n}} ; \boldsymbol{L}_{\boldsymbol{m a x}}\right]$} \\
\hline$H_{A, 1}$ & 2,69 & {$[2 ; 3,4]$} & $H_{B, 1}$ & 3,73 & {$[2 ; 4,5]$} \\
$H_{A, 2}$ & 0,1 & {$[0 ; 0,3]$} & $H_{B, 2}$ & 0,3 & {$[0 ; 0,5]$} \\
$K_{A, 1}$ & 0,0336 & {$[0 ; 0,2]$} & $K_{B, 1}$ & 0,0466 & {$[0 ; 0,2]$} \\
$K_{A, 2}$ & 1,0 & {$[0 ; 2]$} & $K_{B, 2}$ & 3,0 & {$[2 ; 4]$} \\
$k_{e f f, A}$ & 0,1 & {$[0 ; 0,3]$} & $k_{e f f, B}$ & 0,1 & {$[0 ; 0 ; 3]$} \\
\hline
\end{tabular}

Nos resultados seguintes são apresentados gráficos provenientes das execuções usando a metodologia populacional, estabelecendo-se um espaço de busca de $80 \%$ na função que realiza a pequena perturbação (passo de intensificação da busca no algoritmo da Figura 2) e usando os limites apresentados na Tabela 1. Foram utilizados cinco indivíduos no loop externo.

Os gráficos de densidade que descrevem a probabilidade relativa a cada variável em obter o valor esperado e também a dispersão correspondente, são apresentados nas Figuras 4 e 5 . No caso da Figura 4 são apresentados os gráficos de densidade de probabilidade das estimativas sem o uso de informação a priori. Na Figura 5, no entanto, considera-se um valor a priori ao parâmetro $K_{A, 2}$.

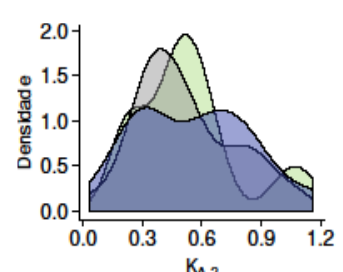

(a)

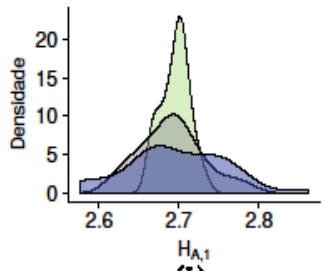

(b)

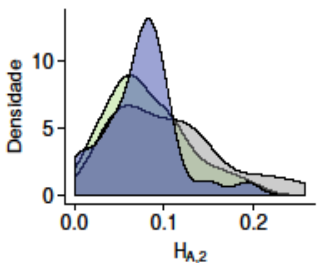

(c)

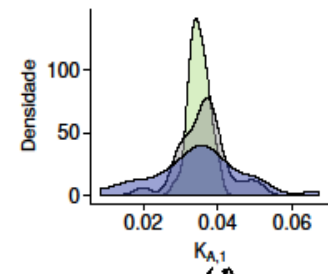

(d)

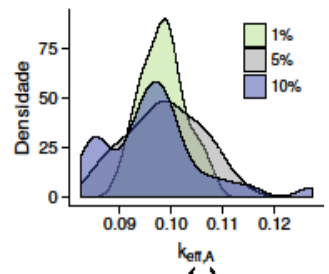

(e)

Figura 4. Gráfico de densidade dos parâmetros usando MLE-PopPCA para minimização da função objetivo

Figura 5. Gráfico de densidade dos parâmetros usando MAP-PopPCA para minimização

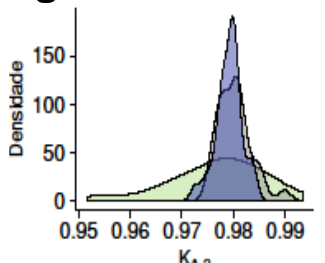

(a)

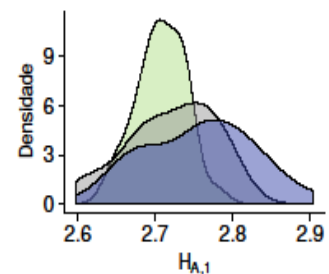

(b)

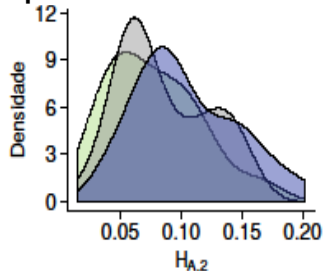

(c)

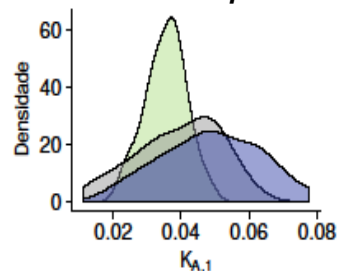

(d)

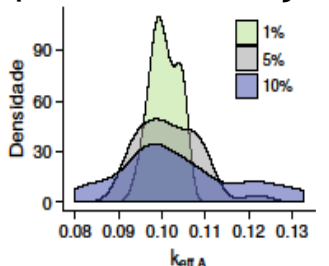

$(e)$

da função objetivo 
Observa-se nos gráficos que ao longo das execuções a melhora se dá apenas no parâmetro em que foi fornecida uma estimativa a priori (Figura $5 \mathrm{a}$ ). Verifica-se ainda que 0 método estocástico usado no MAP apresenta mais importância à informação a priori nos casos em que foram considerados ruídos de $5 \%$ e $10 \%$ nos dados experimentais e com isso, manteve os valores próximos ao valor de referência. $O$ algoritmo atribui mais peso aos dados experimentais quando o ruído experimental é baixo $\left(\sigma_{\mathbf{Y}}=1 \%\right)$ e com isso apresenta amostras ruins.

Com exceção das amostras calculadas com a informação a priori, a análise que se tem dos gráficos apresentados é que o procedimento do MAP resolvido com o PopPCA recuperou melhores estimativas aos parâmetros quando o erro dos dados experimentais correspondeu a 1\%, ou seja, não houve alta taxa de variação das amostras calculadas, em torno do valor de referência.

Na Tabela 2 são apresentadas as médias, desvios padrão e também os coeficientes de variação dos parâmetros da isoterma de bi-Langmuir para a substância menos retida, obtidas com as abordagens MLE e MAP. Pelo coeficiente de variação verifica-se que os maiores valores apresentados correspondem aos parâmetros que possuem relação de simetria na análise de sensibilidade $\left(H_{A, 2}\right.$ e $\left.K_{A, 2}\right)$ e que a informação dada ao parâmetro $K_{A, 2}$ não influenciou no cálculo de boas estimativas para o parâmetro $H_{A, 2}$. Os desvios padrão contidos nos resultados destas simulações possuem duas componentes, uma devida à natureza estocástica dos métodos e outra devida às flutuações nos dados experimentais.

Tabela 2. Média, desvio padrão e coeficiente de variação dos parâmetros $H_{A, 1}$ e $H_{A, 2}$.

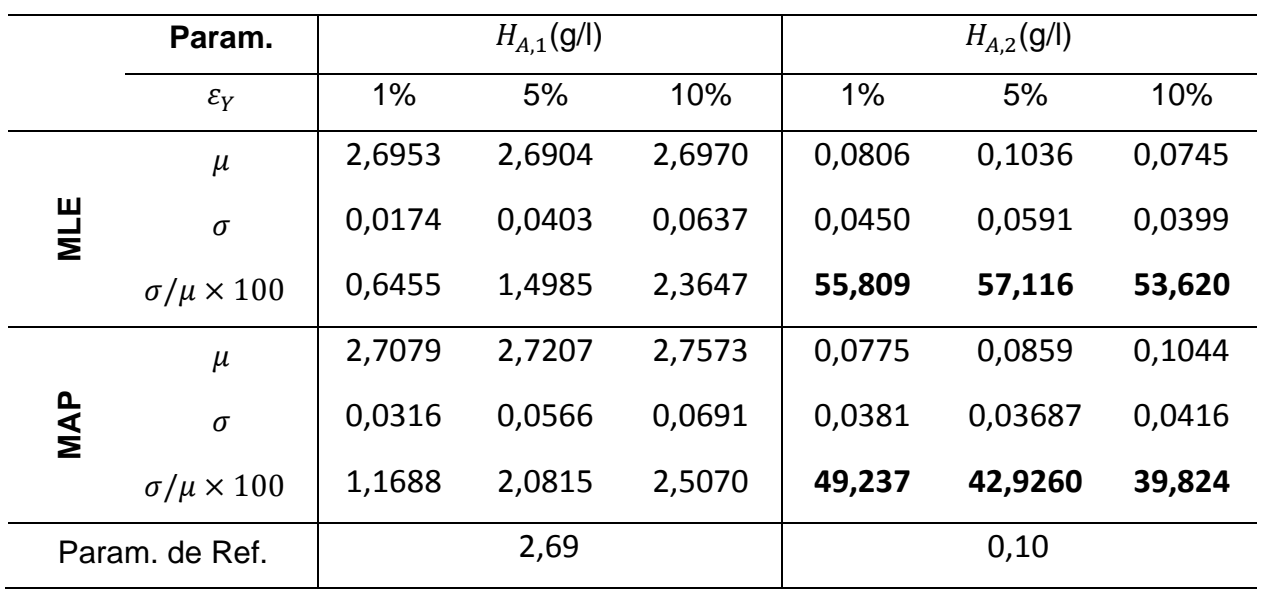


DOI 10.18605/2175-7275/cereus.v10n3p120-135
Oliveira, C.; Lugon Jr., J.; Sacco, W. F.; Knupp, D. C., Silva Neto, A. J. Estimativa de Parâmetros em um Sistema de Leito Móvel Simulado via Algoritmo de Colisão de Partículas Populacional

Na Tabela 3 são apresentados também os valores do desvio padrão e coeficientes de variação para os parâmetros $K_{A, 1}, K_{A, 2}$ e $k_{e f f, A}$, sendo $K_{A, 2}$ sem e com informação a priori.

Tabela 3. Média, desvio padrão e coeficiente de variação dos parâmetros $K_{A, 1}, k_{e f f, A} \mathrm{e}$

\begin{tabular}{|c|c|c|c|c|c|c|c|c|c|c|}
\hline \multicolumn{11}{|c|}{$K_{A, 2}$} \\
\hline & \multirow{2}{*}{$\begin{array}{c}\text { Param. } \\
\varepsilon_{Y}\end{array}$} & \multicolumn{3}{|c|}{$K_{A, 1}$} & \multicolumn{3}{|c|}{$k_{e f f, A}$} & \multicolumn{3}{|c|}{$K_{A, 2}$} \\
\hline & & $1 \%$ & $5 \%$ & $10 \%$ & $1 \%$ & $5 \%$ & $10 \%$ & $1 \%$ & $5 \%$ & $10 \%$ \\
\hline \multirow{3}{*}{$\stackrel{\text { 山}}{\Sigma}$} & $\mu$ & 0,03489 & 0,0362 & 0,0349 & 0,09837 & 0,09868 & 0,0966 & 0,5286 & 0,5163 & 0,5567 \\
\hline & $\sigma$ & 0,0026 & 0,0062 & 0,0121 & 0,00431 & 0,00724 & 0,0097 & 0,2610 & 0,2485 & 0,2871 \\
\hline & $\sigma / \mu \times 100$ & 7,463 & 7,3944 & 34,769 & 4,38456 & 7,3407 & 10,045 & 49,387 & 48,1462 & 51,578 \\
\hline \multirow{3}{*}{$\frac{Q}{\Sigma}$} & $\mu$ & 0,0355 & 0,0391 & 0,0489 & 0,1005 & 0,1017 & 0,1038 & 0,9766 & 0,9802 & 0,9791 \\
\hline & $\sigma$ & 0,0059 & 0,0124 & 0,0142 & 0,0031 & 0,0069 & 0,0138 & 0,0093 & 0,0032 & 0,0025 \\
\hline & $\sigma / \mu \times 100$ & 6,736 & 31,798 & 29,103 & 3,1453 & 6,8539 & 13,305 & 0,9566 & 0,3277 & 0,2554 \\
\hline \multicolumn{2}{|c|}{ Param. de Ref. } & & 0,0336 & & & 0,10 & & & 1,0 & \\
\hline
\end{tabular}

Os gráficos a seguir levam em conta a análise de sensibilidade realizada nos parâmetros da equação que representa a isoterma de equilíbrio. Foram determinados, neste caso, apenas os parâmetros $H_{A, 1}, K_{A, 1}$ e o coeficiente de transferência de massa $k_{e f f, A}$, ou seja, os parâmetros que não apresentaram correlação. As estimativas foram calculadas sem o conhecimento de informação a priori empregando o procedimento MLE. Os resultados são apresentados nos gráficos de densidade de probabilidade na Figura 6.
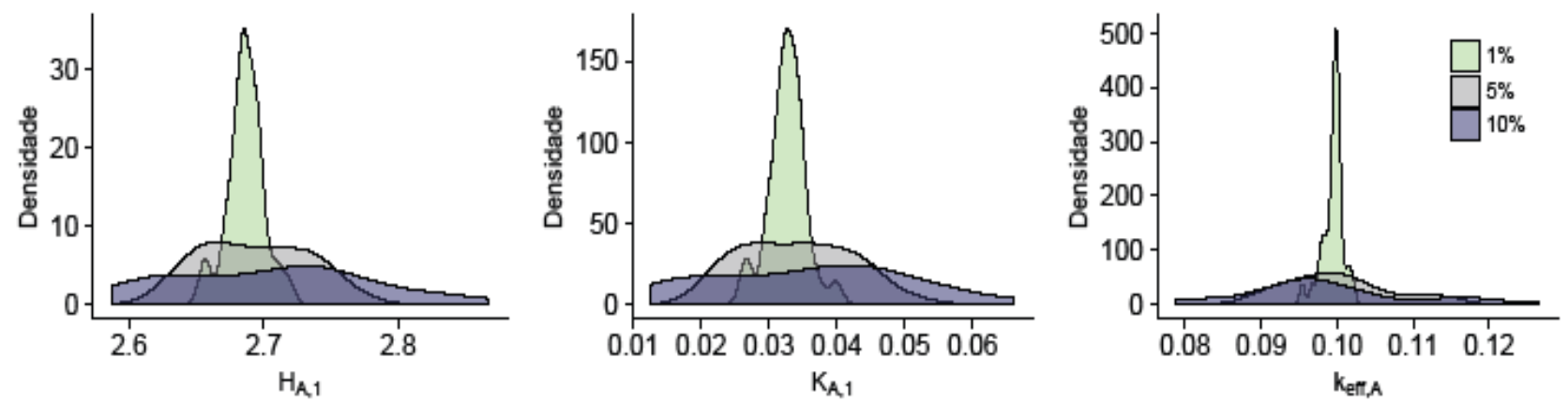

Figura 6. Gráficos de densidade dos parâmetros calculados com o algoritmo PopPCA

Observa-se neste caso que o método foi capaz de produzir boas soluções dentro do limite de busca, principalmente para o caso de menor ruído. Os resultados apresentados para o caso das estimativas de parâmetros correspondentes ao enantiômero B são apresentados na Figura 7 e levam em conta também o resultado da análise de 
sensibilidade. Foi considerado neste caso o cálculo das constantes $K_{B, 1}, K_{B, 2}$ e $k_{e f f, B}$ fornecendo, no entanto, informação a priori ao parâmetro $K_{B, 2}$.
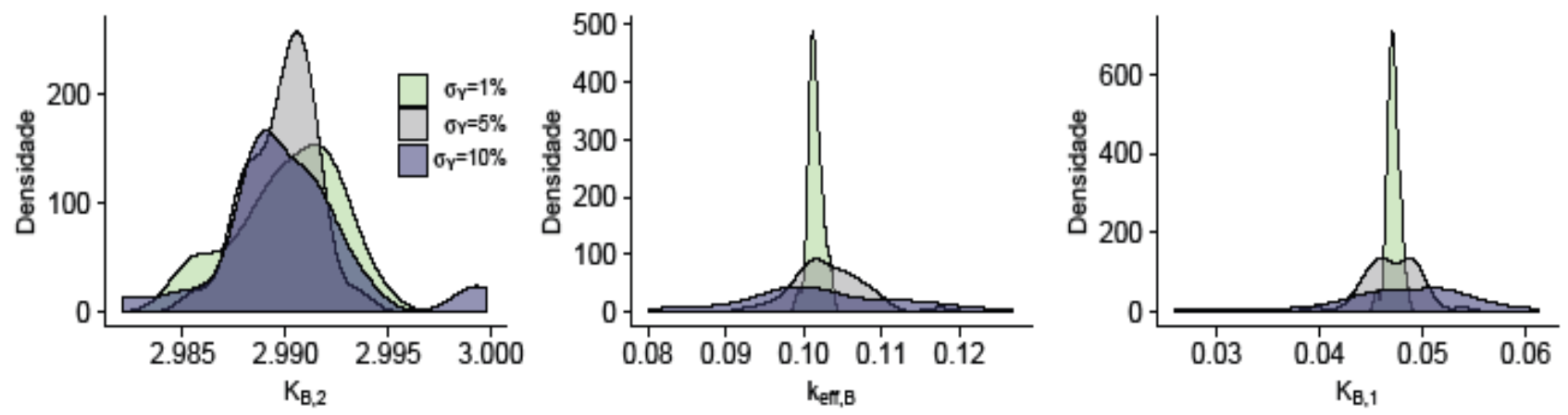

Figura 7. Resultados obtidos pelo procedimento MAP-PopPCA com estimativa a priori ao parâmetro $K_{B, 2}$

\section{CONSIDERACQÓES FINAIS}

Neste trabalho foi aplicado um método estocástico para estimativa de parâmetros em uma unidade de Leito Móvel Simulado. As abordagens utilizadas foram eficazes, produzindo boas estimativas para o ajuste do modelo. Outros experimentos foram realizados considerando o uso de intervalos maiores de busca para o algoritmo PopPCA. Verificou-se, porém, que para intervalos mais largos que aqueles apresentados na Tabela 1, o método não conseguiu apresentar estimativas em torno do valor de referência, havendo maior dispersão dos parâmetros, mesmo quando fornecida uma informação a priori. A limitação com a abordagem empregada é essencialmente o alto custo computacional, requerido para se obter amostras suficientes para avaliar incertezas provenientes de ruído experimental. Isso ocorre quando se aumenta o conjunto de amostras para inferir o desvio padrão dos parâmetros estimados.

\section{AGRADECIMENTOS}

Os autores agradecem o apoio financeiro da FAPERJ - Fundação Carlos Chagas Filho de Amparo à Pesquisa do Estado do Rio de Janeiro, do CNPq - Conselho Nacional de Desenvolvimento Científico e Tecnológico e da CAPES - Coordenação de Aperfeiçoamento de Pessoal de Nível Superior. 


\section{REFERÉNCIAS}

BIEGLER, L.T.; JIANG, L.; FOX, V. G., Recent advances in simulation and optimal design of pressure swing adsorption systems, Separation and Purification Reviews, $n$. 33, p. 1-39, 2004.

GROSFILS, V., Modelling and parametric estimation of simulated moving bed chromatographic processes (SMB). (Tese). Bruxelles Universite, 2009.

HAAG, J.; VANDE WOUWER, A.; LEHOUCQ, S.; SAUCEZ, P., Modeling and simulation of a SMB chromatographic process designed for enantioseparation. Control Eng. Pract., n. 9, v. 921, 2001.

LI, S.; KAWAJIRI, Y.; RAISCH J.; SEIDEL-MORGENSTERN, A., Optimization of Simulated Moving Bed Chromatography with fractionation and feedback incorporating an enrichment step. Computer Aided Chemical Engineering, n. 29, p. 818822, 2011.

LUGON Jr. J., Solução de Problemas Inversos na Adsorção de Proteínas em Interfaces Gás-Líquido e na Secagem em Meio Poroso Únidimensional (Tese) IPRJ/UERJ, 2005.

ÖZISIK, M. N.; ORLANDE, H. R. B., Inverse Heat Transfer: Fundamentals and Applications, Taylor \& Francis, New York, 2000.

PAIS, L. S.; LOUREIRO, J. M. e RODRIGUES, A. E., Modeling, simulation and operation of a simulated moving bed for continuous chromatographic separation of 1,1'-bi-2naphthol enantiomers, Journal of Chromatography A, v. 827, p. 215-233, 1997.

PAIS, L. S.; LOUREIRO, J. M.; RODRIGUES, A. E., Separation of chiral epoxide by simulated moving bed chromatography, Journal of Chromatography A, v. 769, p. 25-35, 1998.

SACCO, W. F.; ALVES FILHO, H; DE OLIVEIRA, C. R. E., A populational particle collision algorithm applied to a nuclear reactor core design optimization. United States: American Nuclear Society - ANS, 2007.

SILVA, E. A. B., Modelagem e Simulação Numérica de uma Unidade de Leito Móvel Simulado. 140f. Dissertação (Mestrado em Engenharia Química) - Universidade Federal de Santa Catarina, 2000.

KAIPIO, J.; SOMERSALO, E., Statistical and Computational Inverse Problems. Springer, New York, v. 160, 2005.

KAWAJIRI Y.; BIEGLER L.T., Optimization strategies for Simulated Moving Bed and powerfeed processes, AIChE Journal, v. 52, p. 1343-1350, 2005. 
KAWAJIRI Y.; BIEGLER L.T., Large-scale optimization strategies for zone configuration of Simulated Moving Beds. Computers \& Chemical Engineering, v. 32, p. 135-144, 2008.

WONGSO, F.; HIDAJAT, K.; RAY, A. K., Optimal operating mode for Enantioseparation of SB-553261 racemate based on Simulated Moving Bed Technology. Biotechnology and Bioengineering, v. 87, p. 704-722, 2004.

YAO, H.; TIAN, Y. C.; TADE, M. O., Using wavelets for solving SMB separation process models. Industrial and Engineering Chemistry Research, v. 47, p. 5585-5593, 2008.

YOUNG, P., The leapfrog method and other "symplectic" algorithms for integrating Newton's laws of motion, p. 1-16, 2014. 